\title{
Bernardo Vieira Bezerra
}

\author{
Estratégia de Oferta em Leilões de \\ Opções de Energia Elétrica
}

Dissertação de Mestrado

Departamento de Engenharia Elétrica

Programa de Pós-Graduação em

Engenharia Elétrica

Rio de Janeiro

Abril de 2006 


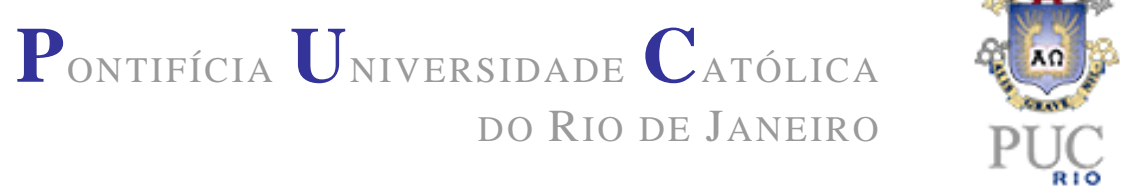

Bernardo Vieira Bezerra

\begin{abstract}
Estratégia de Oferta em Leilões de Opções de Energia Elétrica
\end{abstract}

Dissertação de Mestrado

Dissertação apresentada ao Programa de PósGraduação em Engenharia Elétrica da PUC-Rio como requisito parcial para obtenção do título de Mestre em Engenharia Elétrica.

Orientador: Oscar Porto

Rio de Janeiro Abril de 2006 


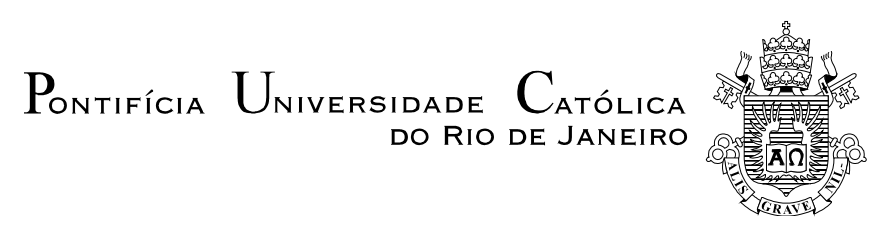

Bernardo Vieira Bezerra

\title{
Estratégia de Oferta em Leilões de Opções de Energia Elétrica
}

Dissertação apresentada como requisito parcial para obtenção do grau de Mestre pelo Programa de PósGraduação em Engenharia Elétrica do Departamento de Engenharia Elétrica do Centro Técnico Científico da PUCRio. Aprovada pela Comissão Examinadora abaixo assinada.

\author{
Dr. Oscar Porto \\ Orientador \\ Departamento de Engenharia Elétrica - PUC-Rio \\ Dr. Mario Veiga Ferraz Pereira \\ PSR Consultoria Ltda \\ Dr. João Carlos de Oliveira Mello \\ Andrade \& Canellas \\ Dr. Armando Matos de Oliveira \\ EL PASO \\ Prof. José Eugenio Leal \\ Coordenador Setorial do Centro \\ Técnico Científico
}

Rio de Janeiro, 05 de abril de 2006 
Todos os direitos reservados. É proibida a reprodução total ou parcial do trabalho sem autorização da universidade, do autor e do orientador.

\section{Bernardo Vieira Bezerra}

Graduou-se em Engenharia Elétrica na área de Sistemas de Apoio à Decisão e em Engenharia de Produção Elétrica na PUCRJ em 2003. Atuou na empresa PSR, onde participou ativamente de estudos relacionados à avaliação financeira de projetos; comercialização de energia; integração dos setores de gás e energia; estudos de planejamento energético e na precificação de contratos de opção de energia (tema deste trabalho).

Ficha Catalográfica

Bezerra, Bernardo Vieira

Estratégia de oferta em leilões de opções de energia elétrica / Bernardo Vieira Bezerra ; orientador: Oscar Porto ; co-orientador: Mário Veiga Ferraz Pereira. - Rio de Janeiro : PUC, Departamento de Engenharia Elétrica, 2006.

119 f. : il. ; $30 \mathrm{~cm}$

Dissertação (mestrado) - Pontifícia Universidade Católica do Rio de Janeiro, Departamento de Engenharia Elétrica.

Inclui referências bibliográficas.

1. Engenharia elétrica - Teses. 2. Leilões de energia elétrica. 3. Otimização estocástica. 4. Programação linear. 5. Risco de contratação. 6. Opção de compra. 7. Precificação de opções. I. Porto, Oscar. II. Pontifícia Universidade Católica do Rio de Janeiro. Departamento de Engenharia Elétrica Letras. III. Título.

CDD: 621.3 


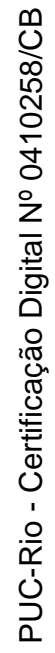

À minha irmã Maria Krishna 


\section{Agradecimentos}

Agradeço à Grazielly Braga de Aquino, minha amada companheira, pelo amor, carinho e paciência.

À minha família. Ao meu pai Luiz Fernardo E. Bezerra, minha mãe Leia Magalhãs Vieira e meus irmãos (Bárbara, Bruno e Maria), além de todos os demais, pelo carinho, amor e apoio neste desafio. Sem eles seria impossível dar este passo.

Ao orientador Mario Veiga Ferraz Pereira, pela oportunidade concedida e confiança nas responsabilidades envolvidas. Agradeço pela excelente orientação em todas as etapas deste trabalho.

Ao orientador Oscar Porto, pela motivação e oportunidades concedidas durante toda minha vida acadêmica.

Ao grande amigo Luiz Augusto, um especial agradecimento. Pelas horas de sono perdidas e dedicadas a todas as fases deste trabalho. Agradeço também pela orientação, pela amizade e pelos incentivos acadêmicos e profissionais.

Ao CNPQ e à PUC-Rio, pelos auxílios concedidos e pelo ótimo ambiente de estudo.

A todos os amigos da Mercados de Energia e PSR Consultoria. 


\section{Resumo}

Bezerra, Bernardo Vieira; Porto, Oscar (Orientador). Estratégia de Oferta em Leilões de Opções de Energia Elétrica. Rio de Janeiro, 2006, 119 p. Dissertação de Mestrado - Departamento de Engenharia Elétrica, Pontifícia Universidade Católica do Rio de Janeiro.

Diversos países vêm utilizando leilões de contratos como mecanismos para induzir à expansão da oferta do sistema elétrico. Em sua grande maioria, o tipo de contrato licitado é um contrato financeiro do tipo "forward". Mais recentemente, o uso de contratos de opção vem sendo utilizado. No caso do Brasil, os contratos de opção de compra de energia elétrica, também conhecidos como contratos por disponibilidade, vêm sendo licitados pelas distribuidoras. Nestes leilões, o vendedor (gerador) participante realiza ofertas simultâneas do prêmio da opção e de seu strike price. Dessa forma, um primeiro desafio é a comparação entre opções com distintos strikes e prêmios. Para um gerador termoelétrico, o desafio subseqüente é como realizar estratégias de ofertas nestes leilões que maximize o retorno do agente, que o torne competitivo e que satisfaça seu perfil de risco. O objetivo desta dissertação de mestrado é desenvolver uma metodologia para determinar a estratégia de oferta de termelétricas em leilões de venda de contratos de opção de compra de energia elétrica. Inicialmente, será apresentado o critério de comparação das opções com distintas características. Em seguida, será estudado o problema de determinar o binômio prêmio de risco e o preço de exercício que devem ser ofertados, visando maximizar a competitividade do projeto no leilão. Adicionalmente, serão analisadas a influência de incerteza no fornecimento de combustível (que introduz incerteza no custo variável de produção) e o perfil de aversão a risco do gerador. Exemplos e estudos de caso serão ilustrados para uma termelétrica bicombustível com incerteza na disponibilidade de gás natural.

\section{Palavras-chave}

Engenharia Elétrica, Leilões de Energia Elétrica, Value at Risk, Otimização Estocástica, Programação Linear, Risco de contratação, Opção de Compra, Precificação de Opções. 


\section{Abstract}

Bezerra, Bernardo Vieira; Porto, Oscar (Advisor). Bidding Strategies in Auctions for Energy Call Options. Rio de Janeiro, 2006, 119 p. MSc. Dissertation - Departamento de Engenharia Elétrica, Pontifícia Universidade Católica do Rio de Janeiro.

The use of a contract auction scheme to induce the electricity system expansion is been carried out worldwide. In most of the cases, the auctioned contract is a financial forward contract. More recently, option contracts are been implemented. In Brazil, energy call options, also known as "availability" contracts, are offered to distribution companies in an auction scheme. On these auctions, the seller (generator) bids both the strike price and the option premium. Consequently, the first challenge is how to compare call options with different strikes and premium. From a thermo electrical generator point of view, the second challenge is how to develop a bidding strategy which maximizes its revenue, competitiveness and taking into account the risk-averse behavior. The objective of this thesis is to develop a methodology for bidding strategies for a thermal plant in auctions for long-term electricity call options. Initially, the problem of comparing call options with different strikes, quantities and premium will be addressed and the solution adopted will be described. We then analyze the optimum bidding strategy, which determinates the premium and strike bids that maximizes the generator competitiveness, taking into account the risk aversion of the generator. Additionally, the cost uncertainty influence will be analyzed (which introduces variable cost uncertainty). Examples and case studies are presented with data from the Brazilian system for a dual-fuel generator with natural gas availability uncertainty.

\section{Keywords}

Electrical Engineering, Electrical power auctions, Value at Risk, Stochastic Optimization, Linear Programming, Contract Risks, Call Options. 


\section{Sumário}

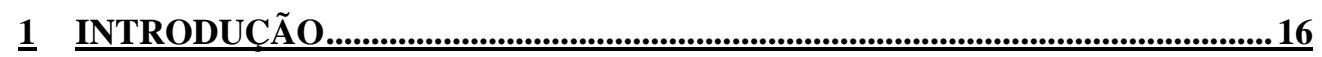

\subsection{O PROCESSO DE REFORMA NO SETOR ELÉTRICO E A GARANTIA DE EXPANSÃO}

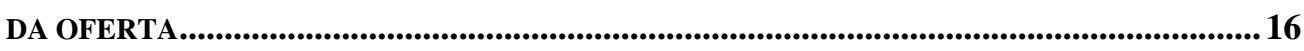

1.1.1 SinAIS DO MERCADO DE CURTO PRAZO E A EXPANSÃo DA OFERTA......................17

1.1.2 CONTRATAÇÃO “FORWARD” DE ENERGIA E A EXPANSÃO DA OFERTA...................18

1.1.3 CONTRATAÇÃO DE “OPÇÕES” DE ENERGIA E A EXPANSÃO DA OFERTA.................18

1.2 CONTRATAÇÃO DE ENERGIA E EXPANSÃO DA OFERTA NO BRASIL ........................ 20

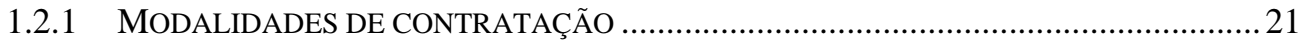

1.2.2 CONTRATOS A TERMO E O LEILÃO DE ENERGIA EXISTENTE....................................22

1.2.3 CONTRATOS DE OPÇÃO E O LEILÃO DE ENERGIA NOVA .........................................23

1.2.4 COMPARAÇÃO DAS OFERTAS EM LEILÕES DE OPÇÕES ...........................................2 23

1.3 ESTRATÉGIAS DE OFERTAS EM LEILÕES DE OPÇÕES DE COMPRA DE ENERGIA. 24

1.3.1 OFERTAS SIMULTÂNEAS DE STRIKE E PRÊMIO ......................................................... 24

1.3.2 INCERTEZA NO SUPRIMENTO DE COMBUSTÍVEL....................................................2

1.4 OBJETIVO DESTA DISSERTAÇÃO ............................................................................... 26

1.5 ORGANIZAÇÃO DA TESE ............................................................................................26

$\underline{2}$ FORMAČ̃̃O DE PREÇOS NO MERCADO DE CURTO PRAZO....................28

2.1 SISTEMAS PURAMENTE TÉRMICOS .......................................................................... 28

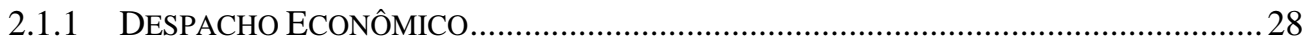

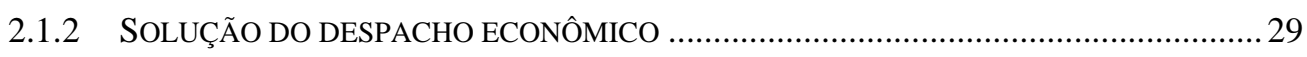

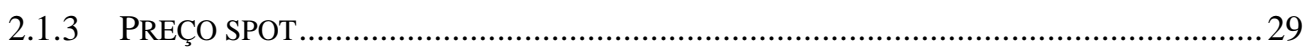

2.1.4 VENDA NO MERCADO DE CURTO PRAZO E RENDA LÍQUIDA ..................................29

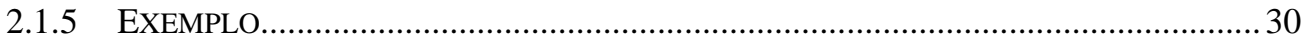

2.2 SISTEMAS HIDROTÉRMICOS .................................................................................... 31

2.2.1 CUSTOS DE OPORTUNIDADE ............................................................................ 31

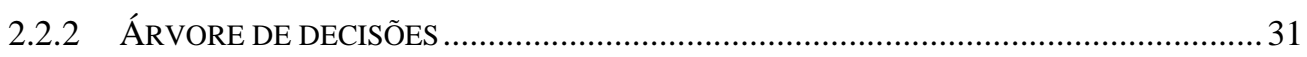

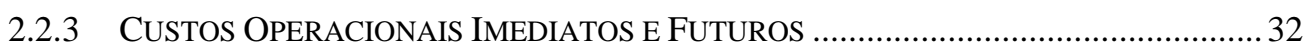

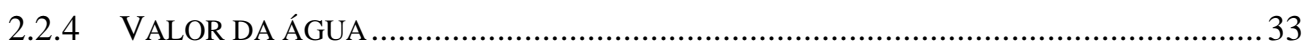


2.2.5 FORMULAÇÃO DO DESPACHO HIDROTÉRMICO PARA UMA ETAPA ............................34

2.2.6 SOLUÇÃO DO PROBLEMA E CUSTOS MARGINAIS........................................................ 36

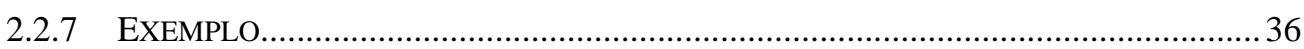

2.3 CÁLCULO DA FUNÇÃo de CUSTO FUTURO ..................................................................38

2.4 VOLATILIDADE DE PREÇOS SPOT E OS SINAIS PARA A EXPANSÃO ..........................41

2.5 VOLATILIDADE DE PREÇOS SPOT E INVESTIMENTOS ............................................42

$\underline{3}$ CONTRATOS A TERMO E CONTRATOS DE OPČÃO ....................................44

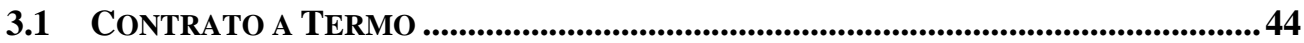

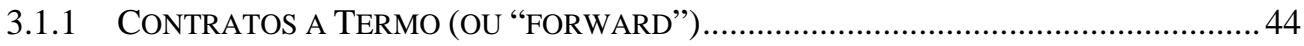

3.1.2 CONTRATOS A TERMO NO SETOR ELÉTRICO ...........................................................4 44

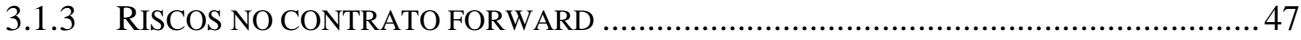

3.1.4 PRECIFICAÇÃO DE CONTRATOS A TERMO ............................................................. 49

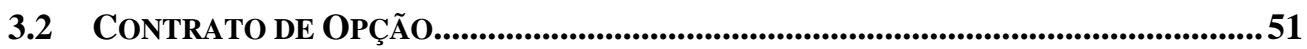

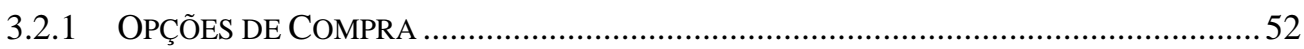

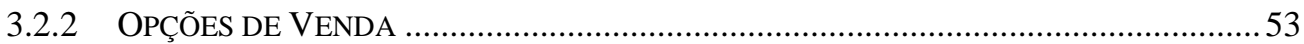

3.2.3 OPÇÕES DE COMPRA NO SETOR ELÉTRICO E OS CONTRATOS POR

DISPONIBILIDADE 54

\section{LEILÕES DE OPCÃO COMO MECANISMO PARA EXPANDIR A OFERTA} 58

4.1 GARANTINDO A SEGURANÇA NO SUPRIMENTO ........................................................58

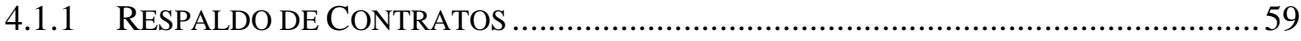

4.1.2 LeILÕES DE ConTRATOS A TERMO E ConTRATOS DE OPÇÃO..................................60

4.2 LEILÕES DE OPÇÃo DE ENERGIA ELÉTRICA: VISÃo GERAL ..................................61

4.2.1 LEILÕES COM OFERTA DE PRÊMIO E QUANTIDADE ................................................. 61

4.2.2 COMPARAÇÃO DAS OFERTAS EM LEILÕES COM OFERTAS DE PRÊMIO E QUANTIDADE 62

4.2.3 ESTRATEGIA DE OFERTA (PRÊMIO DE RISCO) EM LEILÕES COM STRIKE FIXO ......62

4.2.4 LEILÕES COM OFERTA DE STRIKE, PRÊMIO E QUANTIDADE 63

4.2.5 COMPARAÇÃO DAS OFERTAS NO CASO DO BRASIL: O ÍNDICE CUSTO BENEFÍCIO 64

4.2.6 PROPRIEDADES DO ICB.

4.2.7 ESTRATÉGIA DE OFERTAS EM LEILÕES DE OPÇÕES COM OFERTA DE PRÊMIO, STRIKE E QUANTIDADE 
$\underline{5}$ MODELOS COMPUTACIONAIS E VISÃO GERAL DA METODOLOGIA DE

ESTRATÉGIA DE OFERTAS ...................................................................................70

5.1 CENÁRIOS DE PREÇO SPOT E DISPONIBILIDADE DE GÁS ...........................................70

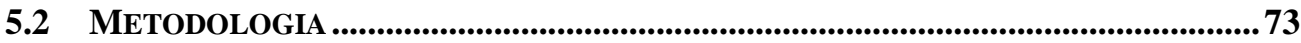

6 ESTRATÉGIAS DE OFERTA EM LEILÕES DE OPÇÕES: UM EXEMPLO 74

6.1 CÁLCULO DO PRÊMIO DE RISCO ................................................................................... 74

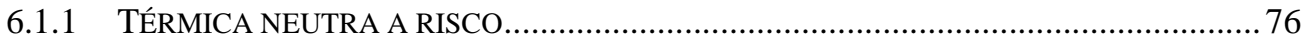

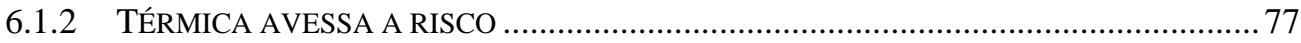

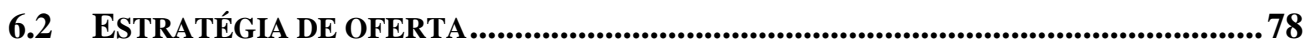

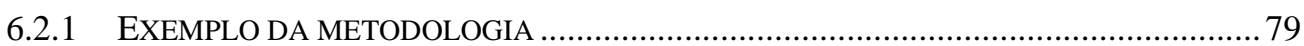

6.3 ESTRATÉGIA DE OFERTA SOB INCERTEZA NO SUPRIMENTO DE COMBUSTÍVEL.80

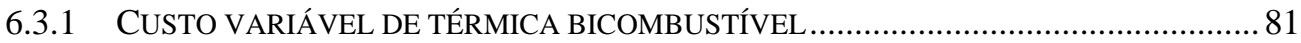

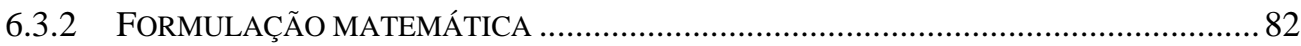

6.3.3 EXEMPLO DA METODOLOGIA PROPOSTA - CASO BICOMBUSTÍVEL ..........................82

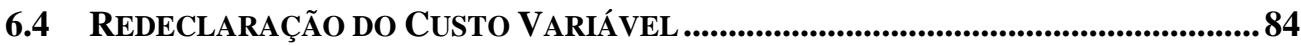

\section{ESTRATÉGIAS DE OFERTA EM LEILÕES DE OPCCÕES: ESTUDO DE}

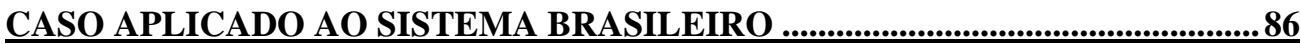

7.1 MODELAGEM DA INCERTEZA NO SUPRIMENTO DE COMBUSTÍVEL ........................86

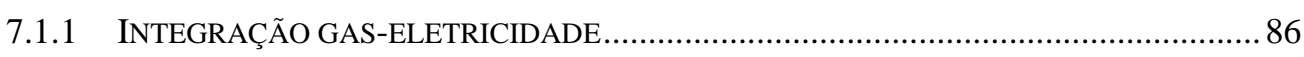

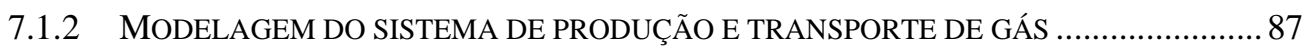

7.2 ESTUdO DE CASO PARA O LEILÃo DE ENERGIA NOVA DE 2005.............................88

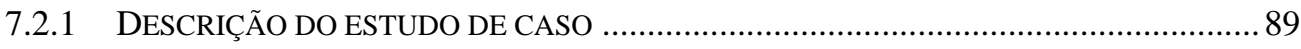

7.3 RESULTADOS.....................................................................................................................90

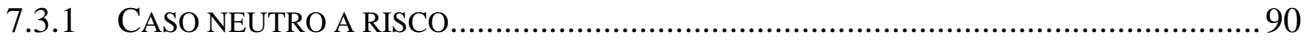

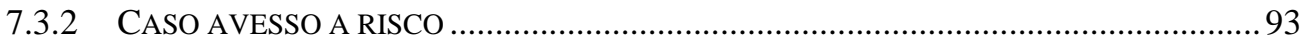

7.3.3 RESULTADOS CONSIDERANDO REDECLARAÇÃO DE CUSTO ....................................95

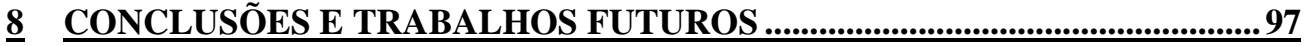

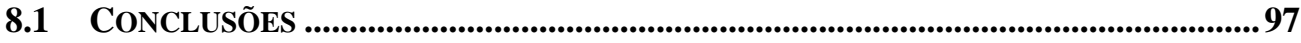

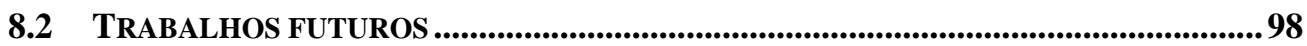




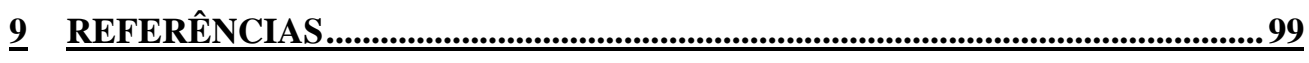

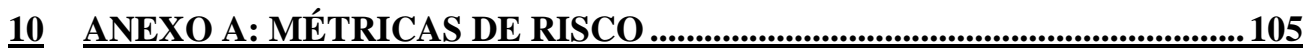

10.1 O MOdelo de OTIMiZaÇão de PoRTfolios de MARKoWITZ....................... 105

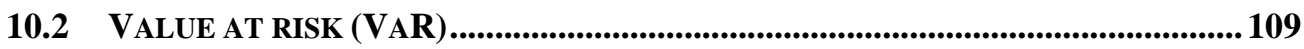

10.3 DOWNSIDE RISK ..................................................................................................... 110

10.4 FUNÇõES DE UTILIDADE .......................................................................................... 113

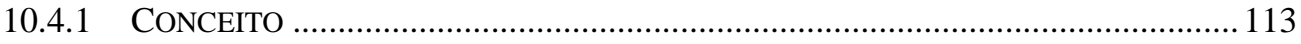

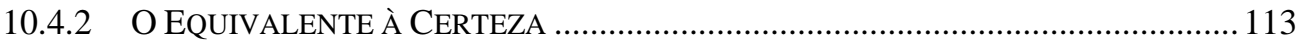

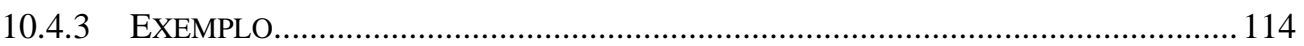

11 ANEXO B: MODELO ESTOCÁSTICO DE DESPACHO DO GÁS - SGAS 116

11.1 O MODELO SGAS ................................................................................................... 116 


\section{Lista de Figuras}

Figura 2-1 - Processo Decisório em Sistemas Hidrotérmicos .............................. 32

Figura 2-2 - Custos (\$) Imediatos e Futuros contra Armazenamento Final $\left(\mathrm{Hm}^{3}\right)$

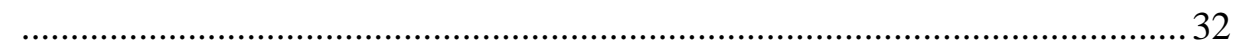

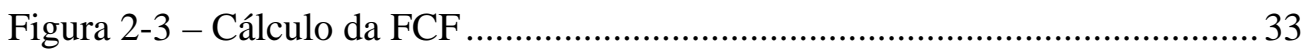

Figura 2-4 - Despacho Ótimo. ......................................................................... 34

Figura 2-5 - Balanço hídrico do reservatório .......................................................... 35

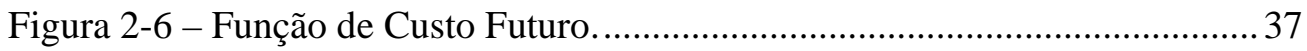

Figura 2-7 - Definição dos Estados do Sistema.................................................. 38

Figura 2-8 - Cálculo da Decisão Ótima por Cenário - Último Estágio.................. 39

Figura 2-9 - Cálculo do primeiro segmento da FCF ........................................... 39

Figura 2-10 - FCF Linear por partes para o Estágio T-1 ….................................. 40

Figura 2-11 - Cálculo do custo operativo para o estágio T-1 e FCF para T-2...... 40

Figura 2-12 - Sistema SE/CO - Custo marginal de curto prazo ............................ 41

Figura 2-13 - Distribuição do Preço “spot” (R\$/MWh)........................................ 42

Figura 3-1 - Distribuição da Receita do Gerador - contrato por quantidade........ 47

Figura 3-2 - Preços “spot” x Nível de Armazenamento (I) - situação seca ......... 48

Figura 3-3 - Preços “spot” x Nível de Armazenamento (II) - situação úmida ..... 48

Figura 3-4 - Lucro com Opção de Compra........................................................... 53

Figura 3-5 - Lucro Com Opção de Venda ............................................................ 54

Figura 3-6 - Distribuição da Receita do Gerador - contrato de opção de compra 57

Figura 4-1 - Lastro x Geração Mínima e Lastro x Preço de Exercício................... 60

Figura 4-2 - Comparação entre ofertas nos leilões prêmio-quantidade ................ 62

Figura 4-3 - Valor esperado do custo operativo x preço de exercício ....................67 67

Figura 5-1 - Cadeia de modelos utilizada na minimização do ICB ......................71

Figura 6-1 - Valor presente da receita operativa da usina ................................... 76

Figura 6-2 - Valor presente da receita líquida - térmica neutra a risco ................ 77

Figura 6-3 - Valor presente da receita líquida - térmica avessa a risco ............... 78

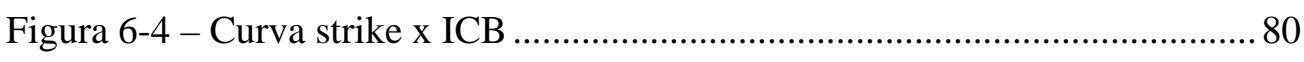

Figura 6-5 - Curva strike x ICB (incerteza no custo)........................................... 83

Figura 6-6 - Strike Ótimo para Cada Nível de Indisponibilidade......................... 84

Figura 6-7 - Receita Extra com Redeclaração ...................................................... 85

Figura 7-1 - Fluxo de Informações SGAS ............................................................ 88 
Figura 7-2 - Preço de exercício ótimo - térmica neutra a risco ............................. 91

Figura 7-3 - Distribuição do valor presente das receitas (neutro a risco) ............. 92

Figura 7-4 - Receita líquida total durante o contrato ............................................ 93

Figura 7-5 - Distribuição do valor presente das receitas (avesso a risco) ..............93

Figura 7-6 - Comparação das receitas - série \#153 .............................................. 94

Figura 7-7 - Preço de exercício ótimo - térmica avessa a risco............................ 94

Figura 7-8 - Distribuição do valor presente das receitas (redeclaração) ................ 96

Figura 10-1 - Distribuição da Geração ............................................................... 106

Figura 10-2 - Medida de Risco ...................................................................... 107

Figura 10-3 - Portfolios de Variância Mínima.................................................... 108

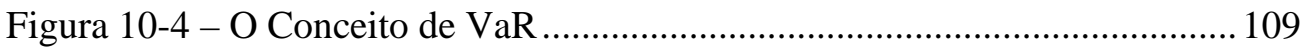

Figura 10-5 - Distribuições de Retorno Assimétricas........................................ 111

Figura 10-6 - Fronteira Eficiente - Downside Risk........................................... 112

Figura 10-7 - Tipos de Função de Utilidade .................................................... 113

Figura 10-8 - Possível Função Utilidade ............................................................ 114

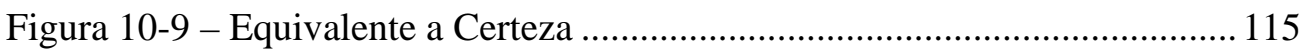

Figura 11-1 - Fluxo de dados do SGAS.......................................................... 117

Figura 11-2 - Grafo representando rede de gasodutos .................................... 118 


\section{Lista de Tabela}

Tabela 2-1 - Características das Termelétricas .................................................. 30

Tabela 2-2 - Características da Hidrelétrica.......................................................... 36

Tabela 2-3 - Despacho Ótimo - Sistema Hidrotérmico....................................... 37

Tabela 3-1 - Cenários de preços spot..................................................................... 50

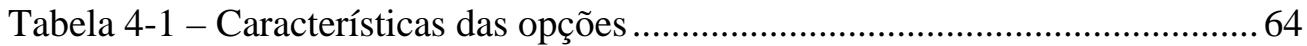

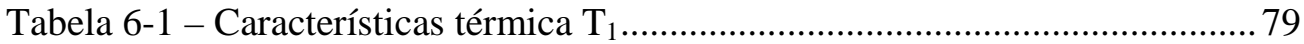

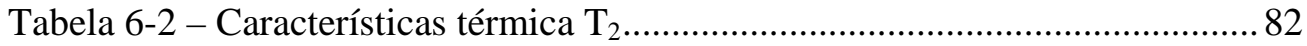

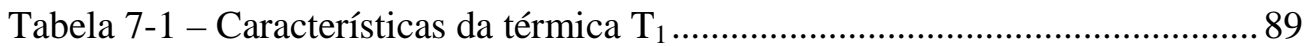

Tabela 7-2 - Resultado (neutro a risco).............................................................. 91

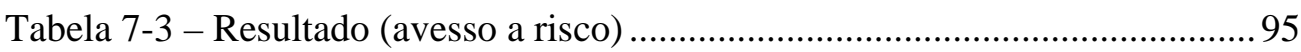

Tabela 7-4 - Resumo dos resultados ………...................................................... 96

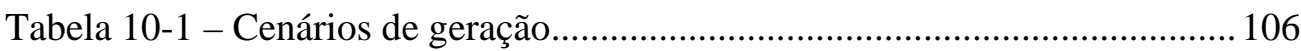




\section{Siglas Utilizadas}

ACL: $\quad$ Ambiente de Contratação Livre

ACR: $\quad$ Ambiente de Contratação Regulado

CCEAR: Contrato de Comercialização de Energia no Ambiente Regulado

CCEE: Câmera de Comercialização de Energia Elétrica

CNPE: $\quad$ Conselho Nacional de Política Energética

CV: $\quad$ Custo Variável

ICB: I Índice Custo Benefício

MME: $\quad$ Ministério de Minas e Energia

O\&M: $\quad$ Operação e Manutenção

ONS: $\quad$ Operador Nacional do Sistema

PMO: $\quad$ Plano Mensal de Operação

VaR: Valor no Risco (Value at Risk)

VP: Valor Presente 